$\S=-1$

\title{
A Review on Employee's Health Monitoring System using IOT
}

\author{
Dr.Ch. N. Santhosh Kumar ${ }^{*}$, Dr. Mohammed Ali Hussain ${ }^{2}$ \\ ${ }^{I}$ Professor, Dept. of CSE, Swarana Bharathi Institute of Science \& Technology, Khammam, Telangana, India. \\ ${ }^{2}$ Professor, Dept. of ECM, Koneru Lakshmaiah Education Foundation, Guntur Dist., Andhra Pradesh, India. \\ *Email: santhosh.ph10@gmail.com
}

\begin{abstract}
Due to the automation trends going on rapidly on these days in major organizations employees are constantly facing severe stress regarding their jobs. This insecurity levels in them is leading to several health problems some of which are to be monitored and proper medical care should be taken. Due to the work stress and lack of leisure's time many Employee's are postponing their health check-ups and facing major health issues. These health issues like blood pressure, heart diseases, diabetes, pneumonia etc., can be kept at checking levels if they are regularly monitored and avoiding future problems. Due to these health problems employees are not able to do their work as expected. So if the organizations take a major initiative regarding in this point of view by keeping a regular check on employees health and there routine medical check-ups by sending periodic alert messages to the corresponding employee reminding about their check-ups. In this Paper we are trying to design a software Application which enables the organization to provide necessary health check-up facilities to their employee's by collecting the medical information from each employee while joining the organization. It can also track employee health condition during his travel by using GPS. It would be of a great help to every employee. The organization collects medical information regarding the employee's making it mandatory process and storing the data in database (PHR),
\end{abstract}

Keywords: PHR, Sensors, SMS and GPS.

\section{Introduction}

\subsection{Personal Health Record (PHR)}

Personal Health Record is a branch about data framework remarkably intended to help the association and engineering of the health related issues. The reasons for this format is of major help for basic activity for viewing and authorizing the therapeutic issues and screen advance help towards providing aid in achieving health goals, helping thus to scramble toward screen and authority the cut-off of retouching office with utilizing choice help pointer. Similarly, PHR besides help the ace and medicinal staff to enhance health organizations. A remedial or health improvement schedule is a touch about PHR through which given by relationship to their experts. It is a worker progress advantage design created or kept up by a business, an operator association or both. This schedule gives healing consideration to operators and their people especially or into security, repayment. The reason for this schedule is to guarantee a sound effort condition. Also, the health and prospering of representatives are fundamental in keeping up the specialists persuading, safe and unequivocally affected to achieve the association mission what's more, vision. Along these lines, all affiliations having their own particular wellbeing schedule accommodated their operators.

The Electronic Medical Record (EMR) is a one example about HMIS. It is by uprightness of the cut-off about EMR format is to help the association of patient remedial records. In Malaysia, there are several patching office utilizing EMR as a medium to oversee and screen understanding healing record and help experts and remedial gatekeepers in watching patients' pharmaceutical, for example, the prescriptions or solution captured by patients amidst.. Authority's office are among pro's workplaces. Regardless, the EMR is basically utilized as a bit of pro's workplaces thusly this format can't be gotten to by individuals other than experts, restorative watchmen and retouching office affiliation. Subsequently, the EMR isn't sensible to be utilized as a bit of affiliations.

In this paper, Employee Medical History Management[23] and Checking Scheme was required into make an networked database of labourers restorative past events for relationship of director, make coordinate information[24] exchange between business, ace and social affirmation applying good position and plan data joining of patient record[11] complete cobweb affiliations. Thusly, this format can pound the piece of actual steady report scheme. In the running with allocate, paper depicts the format which can be used.

\subsection{GPS}

We already know that the word GPS comes from global positioning system its position is calculated by using latitudes and longitudes. Generally we all know that gps is used to track the exact location anywhere in the map. This system may also useful for communication process among the two points. The majority of following architecture are form by bestow GPS. This is uncommonly direct and pitiful. Following formats are generally used by task force managers for following a vehicle zone, steering 
and others. GPS device receives the coordinates from the GPS satellite for each and every second, along with the time and date and also calculates the device's geographical position. GPS provides maximum accuracy of the required data. Using suitable software, the device may display the position on a map, and it may offer directions too if required.

\subsection{Sensors}

\subsubsection{Heartbeat sensor}

A pulse sensor is an instrument utilized for estimating the heart in the beat sensor relies upon the govern of photo plethysmography. In case of usages where heart beat percentage is to be watched, the arranging of the thumps is crucial. The surge of plasma number is picked by the estimate of heart pulsates and as luminous is devoured by plasma, the banner thumps are corresponding to the heart beat thumps.

\subsubsection{Temperature sensor}

Temperature is a standout amongst the most ordinarily estimated parameter on the planet. They are utilized as a part of your day by day family gadgets from Micro wave, coolers, AC to all fields of building. Temperature sensor essentially measures the warmth/cool created by a protest which it is associated. It at that point gives a relative protection, current or voltage yield which is then estimated or prepared according to our application. LM35TemperatureSensor which is a semiconductor placed sensor. LM35 is an incorporated similarity temperature sensor whose electrical yield is relative to Degree Centigrade. LM35 Sensor does not require any outer adjustment or trimming to give normal exactness.

\subsubsection{Accelerometer sensor}

A champion among the most understood inertial sensors is the accelerometer, a dynamic sensor prepared for a tremendous extent of recognizing. Accelerometers are available that can measure accelerating in one, two, or three orthogonal tomahawks. The fundamental manage of movement behind the MEMS accelerometer is the migration of a little check mass scratched into the silicon surface of the planned circuit and suspended by little shafts.

\section{Motivation and Related works}

\subsection{PHR System on IoT Module}

We have depicted the qualities of a PHR along with the people's part in making and keeping up this record vault. A PHR[12] framework works as a locally established door ready to deal with a whole family's PHR while additionally supporting assembling and putting away crude therapeutic information pushed from the doctor's facility and individual health gadgets. Furthermore, a PHR framework ought to give secure, consistent information imparting to other medicinal services specialist co-ops through an open cloud[16]. To meet these prerequisites, the proposed PHR framework is executed on Samsung's ARTIK 10 IOT module. As a section of IOT Cloud[15] biological community, it furnishes high similarity with ARTIK Cloud, general society cloud utilized for this task. In addition, the module's wide assortment of network A exemplary of our clinic data framework. The HIS comprises of a few open-source ventures: FHIR server, 'Shrewd on Genomics', and an email module.

Bluetooth, Zig-Bee, Wi-Fi, and so on.)enables it to help a wide range of individual health gadgets. As the ARTIK module underpins high speed interfaces, it is appropriate to store crude EMR information through expanded capacity limit. Also, the ARTIK 10 module has committed security equipment and scheduling segments which empower us to safely speak with other individual health gadgets and open mists. As an individual-driven PHR archive, the PHR supervisor web application keeps running on a Tomcat server, getting clinical information from doctor's facility and health information[20] from cell phones. The PHRs are put away in the inward database utilizing the HL7 standard notices. The PHR framework can likewise push health information[25] to healing centres with the goal that clinicians can screen any of their patients' health influencing life logs. The PHR framework occasionally transfers the PHRs to the general population cloud for reinforcement what's more, offering to outsiders. With the interconnected framework placed over IOT module, clients can physically possess and oversee relatives' PHRs in their home.

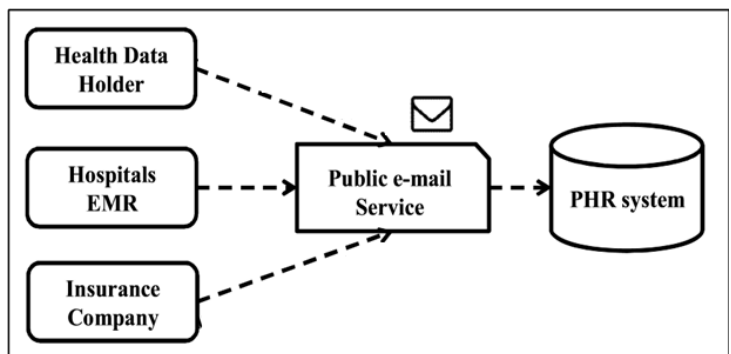

Fig 1:A "push" strategy for health information correspondence. Health information suppliers speak with people by means of their open mail servers through a mechanized procedure.

\subsection{Public Cloud APIs for Private Data Sharing}

One of the undertaking objectives was to permit sharing of client information with outsiders. IOT Platforms can have apparatuses to help this, be that as it may, they put security dealing with immovably responsible for the outsider. We rather longed for the client to be better educated and to have a more granular authority over the information they share, while muddling superfluous recognizing client data. To interface clients and outsiders, we made an entry for the IOT Platform to go about as a 'go-between' or API wrapper. This entryway would be enlisted as an application with the IOT Stage, and would utilize open APIs [17] to accumulate client information. third parties wishing to get to client data would make asks for through this gateway, the entrance would rehash this demand to the IOT Platform, alter the reaction to be in agreement with the client's security and restore this data to the third party. On this entry, outsiders could enlist as an information [26] gathering benefit, demonstrating what kinds of client and gadget information they wish to gather. At the point when clients endeavour to join this administration, the entryway will demonstrate to the client what information they will impart to this benefit, which clients must consent to or not. Just the settled upon client information is then imparted to the outsider.

This entry was considered important because of the conduct of IOT stages where once a client has conceded access to a third party, that outsider has full access to client data and any other information sources that had been asked. (e.g. Envision a savvy that accumulates heart rate and GPS. This gateway permits clients to impart to the outsider just the heart rate information instead of expecting to share both.) Also, the entry gives obscurity by making one of a kind false names for clients and information sources that associate with a benefit. The false name permits a client and all their associated gadgets to remain a durable entire for this administration, however not effectively conspicuous when associated with another administration. This is a powerless type of anonymization, as specific types of distinguishing data will essentially stay in place (GPS information, as an illustration). The entryway allows client caution in what they share, yet this still remains a worry. More grounded types of anonymization (kanonymization strategies, and so forth.) can require top to bottom systems and information, and can require 'settling' the dataset before sharing it. This usefulness could be added to this gateway 
later on, if esteemed vital. Usage of associating the outsider with Jumbled User Data works consequently:

(1) User agrees to offering their information to Platform by means of IOT Platform

(2) outsider joins with entry, enrolling the kinds of client data and other information sources they will gather.

(3) User is coordinated to outsider's posting on the entry, and chooses whether to join subsequent to review the asked information.

(4) The outsider would now be able to ask for client information through the entry, and the gateway will get the asked for information that is put away on the IOT Platform, expel or jumble the information not consented to by the User, and return it to the third party.

\section{Proposed Work}

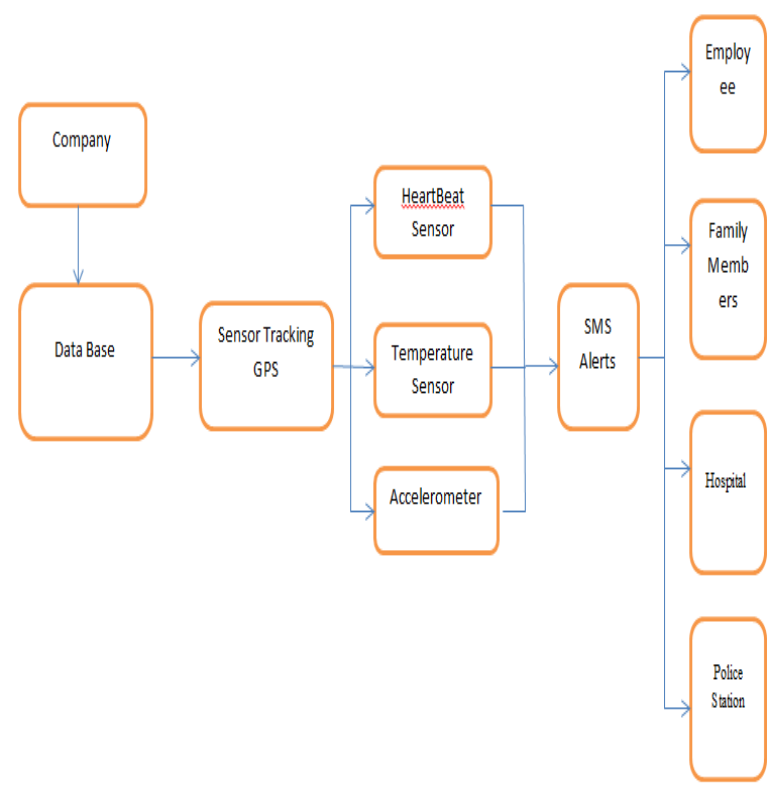

Fig 2: Block diagram of the proposed software Application

\section{1) Tracking Location Using Google Map}

We can find the correct area of the patient[13] and the place of mishap event utilizing the estimations of Longitude and Latitude got from GSM and GPS. Therefore can spare time to give medicinal help. In additional[9] facility which helps tracking of the location of accident by using GPS and GSM modules through which we can get the values of latitudes and longitudes. To further enhance this we used the software of Google maps to track the exact location where the accident site is and thus we can send information to the Police station, and also help in providing emergency medical help by informing the nearby hospitals to send ambulance. Even the family member of the driver will get the information about the accident occurrences and the location. All this information is sent through Mobiles technology in the form of SMSs.

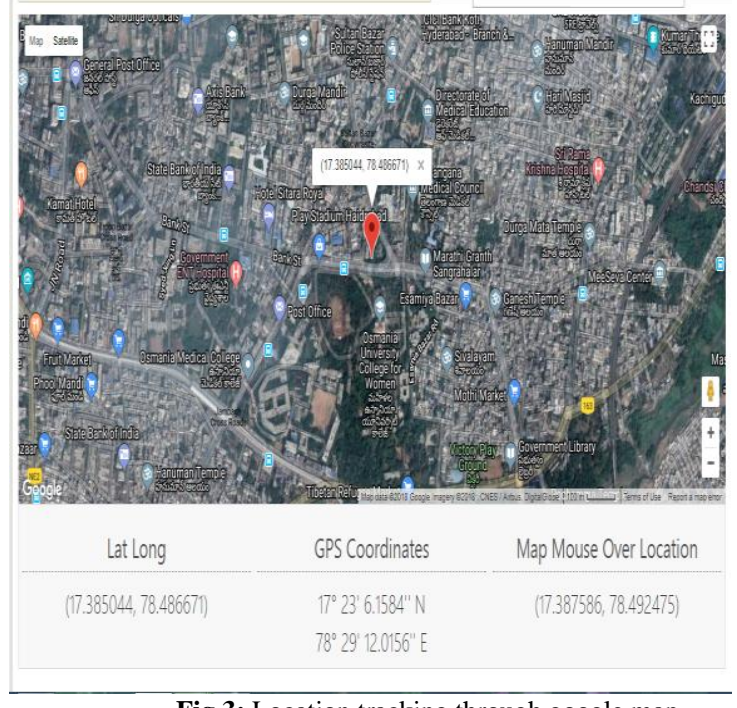

Fig 3: Location tracking through google map

\section{2) SMS Sent to Android Mobile:}

By utilizing HTTP requests and responses on the off chance that it conceivable, on the grounds that this is a straightforward method to send information from an application to another one. Since Java has local technique calls to send HTTP asks for, HTTP gives an extraordinary method to send SMS notices to remote versatile phones. Java can be utilized to send SMS notices. HTTP is utilized to build up association between the Java application and the SMS Gateway. On the off chance that the qualities are unusual when contrasted with pre-stacked esteems in the scheduling, at that point these qualities are sent a SMS to android mobiles to alarm the patient , specialist, police, and relative. Hence helping them to take expected measures to spare patients.

\section{Literature survey}

In this paper recommended that property placed framework [1] is a successful answer for safely overseeing data in tremendous, disseminated frameworks. Yet, in the conventional exemplary, this framework can be accomplished just when single put stock in specialist (TA) is utilized as a part of the framework. This implies the client and server prerequisite in a confided in space. After all the TA can get to all the scrambled documents, it brings about crucial security issue. Single TA additionally makes a heap chokepoint. This makes a hidden danger to the security.

Primary-arrangement quality placed encryption is adjusted type of the Trait Placed Encryption. The paper [2] proposed primaryapproach trait placed encryption (KP-ABE) procedure to defeat the restriction of conventional exemplary. The new information get to authority system i.e. Quality placed encryption (ABE) strategy was presented which comprise of primary-arrangement property placed encryption (KPABE). In this plan, every client will be attributed to an entrance format that will characterize which sort of figure notice the primary can unscramble. The mystery primary is characterized to mirror the entrance format. So client will be equipped for unscrambling a figure content if and just if the information characteristic fulfils that client's entrance format. The KP-ABE is helpful for guaranteeing the fine droped get to authority to information display where it can quickly indicate any information exemplary can be gotten to by which client and what are the capacities they can assassinate up yonder. In any case, this procedure has the drawback that the information proprietor is additionally a put stock in expert (TA). In the event that this strategy is connected to PHR framework with numerous clients and information proprietors, it would be insufficient as every client would get different primary from various proprietors, 
regardless of whether the primary contains a similar arrangement of qualities.

Another changed type of $\mathrm{ABE}$ called Figure content approach Property placed Encryption (CP-ABE) was presented by sahai et al [3]. It enables the information proprietor to encode the information in light of an entrance arrangement, which will be founded on the characteristics of the client or information. Along these lines, the unscrambling is conceivable when the discharge primary is the one comparing to get to authority strategy. The primary thought of $\mathrm{CP}-\mathrm{ABE}$ is that the client mystery primary is related with an arrangement of traits and each figure content will be installed with an entrance format. The client can unscramble the notice just if the clients property matches with the entrance format of the figure content. This technique has pick up that the outsider disjoin won't approach on the real information, decoding will be conceivable just when the mystery primary is coordinated with get to arrangement characterized on properties, and each client is required legitimate approval to get to the information. And furthermore it evacuates the requirement for knowing the character of the patients for giving access give. $\mathrm{CP}-\mathrm{ABE}$ enhances the hindrance of KPABE that the scrambled information can't pick who can unscramble it. The primary issues with respect to this procedure are: Client appointment is testing, Access right of the client can't be adequately overseen by the proprietor, Client properties are sorted out legitimately as a solitary set, so clients can just utilize all conceivable mix of characteristics in a solitary set issued in their primary fulfill arrangements that are just upheld by unscrambling primary.

The multi-expert property placed encryption plot [4] is a headway of trait placed encryption is a numerous quality specialists for taking care of the distinctive arrangement of clients from different spaces. In Personal Health Record framework the clients will be shape distinctive area like doctor, companion or relative from individual relations and different clients from protection organization as well. So every client will have diverse access authority instrument in view of the connection with patient or proprietor. The MA-ABE plan will profoundly lessen the primary administration issues and upward[5]. The guarantee and protection worries of cloud placed PHR framework can be tended to by incorporating progressed cryptographic methods, for example, MA-ABE to PHR framework. In the interim patient increase complete authority approach up to there PHR records and can arranged entry benefit to chose information clients. Hence the dynamic arrangement administration demonstrate is bolstered by this procedure. With higher security and protection for PHR, the current MA-ABE could be wasteful to illuminate the more elevated amount issues [6].

\section{Sample result:}

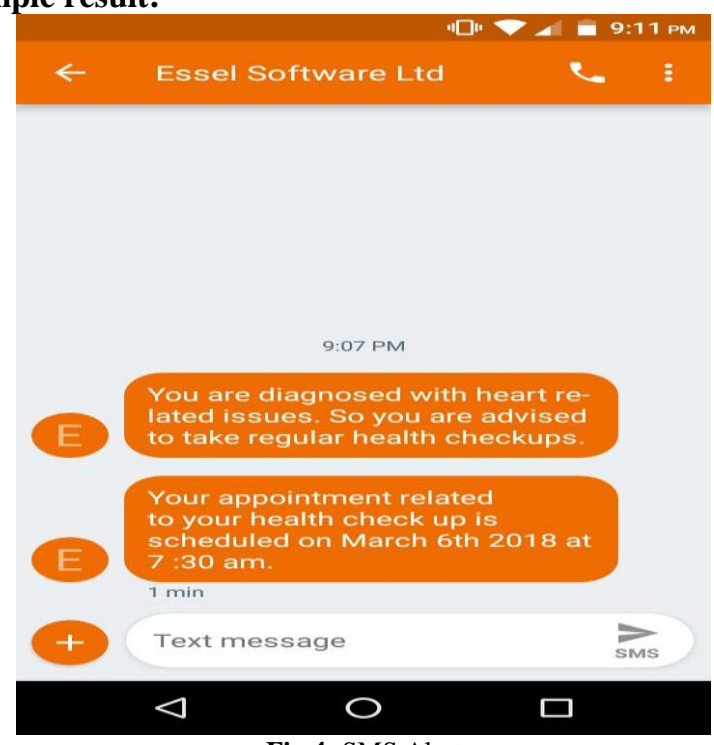

Fig 4: SMS Alert

\section{Conclusion}

This system empowers the supervisor in managing the issues related to necessary treatment methods and moreover to screen the health level of their organization workers. Furthermore, panel clinics can cache and recoup patient or staff therapeutic records from the main database of this system. In this way, the system can urge the board facilities to give proper treatment. The site furthermore goes about as medium for information exchange between supervisor, specialists and board facilities. The execution of worker individual health record administration framework can be of great help to the Chief and board offices in ensuring their staff in getting the best restorative health checking. The board office makes it mandatory that every one of them agrees to implement the necessary methods for maintain every employee's personal health record and also keep monitoring by this efficient software system which is definitely not hard to work and help the business production by screening health level of their employees. The method of sending SMS alerts using cloud API key makes it easy to send in bulk or individually. Further, we can include some more highlights which can help representatives in the organizations to work with the testing angles.

\section{References}

[1] M. Pirretti, P. Traynor, P. McDaniel, and B. Waters, "Secure Attribute Placed Systems," Journal of Computer Security, vol. 18, no. 5, pp. 799-837, 2010.

[2] V. Goyal, O.Pandey, A. Sahai, and B. Waters. "Attribute- Placed Encryption for Fine-droped Access Authority of Encrypted Data", Proc. 13th ACM Conf. Computer and Comm. Security (CCS '06), pp. 89-98, 2006.

[3] Bethencourt, A. Sahai, and B. Waters, "Ciphertext-policy Attribute Placed encryption,” in IEEE S\& P '07, 2007, pp. 321 334.

[4] Huang Lin, Zhenfu Cao, Xiaohui Liang, Jun Shao,"Secure Threshold Multi Authority Attribute Placed Encryption without a Central Authority," INDOCRYPT, 2008, pp. 426-436.

[5] Ramasamy S, Vahidhunnisha, "Survey on Multi Authority Attribute Placed Encryption for Personal Health Record in Cloud Computing", International Journal of Latest Trends in Engineering and Technology, November 2013, pp.223- 229.

[6] Neetha Xavier, "Security of PHR in cloud computing using ABE technique", International Journal of Communication and Computer Technologies, volume 01-No.72 issue: 07, Nov 2013, pp. 265-269

[7] Dawson, J. (10 November 2007). Employer Sponsored Personal Health Record (PHR) Systems, Poster presented at American Medical Informatics Association 2007 Annual Symposium, 10 Nov. 2007, Chicago, Illinois.

[8] Goldman, J. (2007), "Personal Health Records:Employers Proceed with Caution," California HealthCare Foundation Issue Brief, Jan 2007, 1-4

[9] U.SaiNishita, K.Anusha, K.RamyaSriSai, Dr. Mohammed Ali Hussain Health Care Monitoring and Accident Tracking System Placed on Location Awareness International Journal of Engineering \&Technology(2018).

[10] Galvin, R.S. and Delbanco, S. (2006). Between a Rock and a Hard Place: Understanding the Employer Mind-Set," Health Affairs, 25(6), 1548-1555.

[11] Smolij K, Dunn. K.: 'Patient health information management: searching for the right exemplary', Perspect Health InfManag, 2006, 12, pp. 3-10

[12] Tang, P., Ash, J., Bates, D., Overhage, M. and Sands,D. (2006) Personal Health Records: Definitions, Benefits, and Strategies for Overcoming Barriers to Adoption,Journal of the American Medical Informatics Association,

a. 13(2) 121-126.

[13] Ramsaroop, P. and Ball, M.J. (2000). The bank of health.” A exemplary for more useful patient health records.MD Computing 17(4) 45-48. 
[14] Markle Foundation (2005). "Attitudes of Americans Regarding Personal Health Records and Nationwide Electronic Health Information Exchange." October 2005.

[15] E. Ekonomou, L. Fan, W. Buchanan, and C. Thüemmler, "An integrated cloud-placed healthcare infraformat," Cloud Computing Technology and Science (CloudCom), 3rd International Conference, IEEE, 2011.

[16] M. Deng, M. Petkovic, M. Nalin, I. Baroni, "A Home Healthcare System in the Cloud--Addressing Security and Privacy Challenges,"CLOUD, International Conference on, IEEE, pp. 549-556, 2011.

[17] S. Tyagi, A. Amit and M. Piyush, "A conceptual framework for IoTplaced healthcare system using cloud computing," Cloud System and Big Data Engineering, International Conference, IEEE, pp. 503-507, 2016.

[18] P. C. Tang, J. S. Ash, D. W. Bates, J. M. Overhage, and D. Z. Sands, "Personal health records: definitions, benefits, and strategies for overcoming barriers to adoption," JAMIA, 13.2, pp. 121-126, 2006.

[19] Markle Foundation, "Connecting for Health," The personal health working group, Final report, July 2003.

[20] J. Walker, E. Pan, D. Johnston, J. Adler-Milstein, D. W. Bates, and B.Middleton, "The value of health care information exchange and interoperability," Health affairs, 24, W5, 2005.

[21] J. G. Anderson, "Social, ethical and legal barriers to ehealth,"International journal of medical informatics, 76.5, pp. 480-483, 2007.

[22] S. Pais, P. Dave, and H. Yunfeng, "Suitability of Fast Healthcare Interoperability Resources (FHIR) for Wellness Data," Proceedings Ivan Pavlovic, et al, "The Web-Placed Medical Record System to Support Clinical Trials", Faculty of Engineering, University of Ljubljana.2004.
[23] Miguel Cruz A. et al, "Website for Hospital Technological Management System Support", Centro de Bioingenenieria (CEBIO), Ciudad Habana,Cuba.2003.

[24] Developing Health Management Information Systems: A Practical Guide For Developing Countries, World Health Organization (WHO),

a. 2004.

[25] Health Management Information Systems, IDS Health \& Development Information Team, DFID Health Resource Centre.

[26] Mona Khandhar and Amarjit Singh, "Health Management Information Systems (HMIS)", Department of Health \& Family Welfare, Gandhinagar,Gujarat.2008. 